Original article

\title{
Protozoan and bacterial pathogens in tick salivary glands in wild and domestic animal environments in South Africa
}

\author{
M. Berggoetz ${ }^{\mathrm{a}, *}$, M. Schmid ${ }^{\mathrm{a}}$, D. Ston ${ }^{\mathrm{a}}$, V. Wyss ${ }^{\mathrm{a}}$, C. Chevillon ${ }^{\mathrm{b}}$, A.-M. Pretorius ${ }^{\mathrm{c}}$, L. Gern ${ }^{\mathrm{a}}$ \\ a Institut de Biologie, Laboratoire d'Eco-Epidémiologie des Parasites, University of Neuchâtel, Emile Argand 11, 2000 Neuchâtel, Switzerland \\ ${ }^{\mathrm{b}}$ Maladies Infectieuses et Vecteurs: Ecologie, Génétique, Evolution, Contrôle (MIVEGEC; UMR 5290 CNRS-IRD-Universités Montpellier I et II), Montpellier, \\ 911 Avenue Agropolis, BP 64 501, 34394 Montpellier cedex 5, France \\ ${ }^{\mathrm{c}}$ Department of Health Sciences, Faculty of Health and Environmental Sciences, Central University of Technology, Free State Province, Bloemfontein 9300, \\ South Africa
}

\section{A R T I C L E I N F O}

\section{Article history:}

Received 10 July 2013

Received in revised form 10 October 2013

Accepted 14 October 2013

Available online $\mathrm{xxx}$

\section{Keywords:}

Ticks

Tick-borne pathogens

African wildlife

Livestock

Coinfections

Theileria

Babesia

Ehrlichia

Anaplasma

\begin{abstract}
A B S T R A C T
A total of 7364 ticks belonging to 13 species was collected from 64 game animals (belonging to 11 species) and from 64 livestock animals (cattle and sheep) living in close vicinity at 6 localities in 3 South African Provinces (Free State, Mpumalanga, and Limpopo). The geographic distribution of all tick species was congruent with the literature except for Haemaphysalis silacea. From each infested host, a maximum of 10 males and 10 females of each tick species were dissected to isolate the salivary glands. Salivary glands were screened for tick-borne pathogens using polymerase chain reaction followed by reverse line blotting and sequencing. This approach allowed us to evaluate the exposure of wild and domestic hosts to tick-borne pathogens in their respective environments. Among the 2117 examined ticks, 329 (15.5\%), belonging to 8 species, were infected and harboured 397 infections. Among those, $57.7 \%$ were identified to species level and were assigned to 23 pathogen species of the genera Babesia, Theileria, Anaplasma, and Ehrlichia. In 3 out of 6 localities, salivary glands from ticks infesting wild ruminants displayed significantly higher infection prevalence and pathogen mean density than salivary glands from ticks infesting livestock animals. Four piroplasm species [Theileria bicornis, Babesia sp. (sable), Theileria sp. (giraffe), and Theileria sp. (kudu)] were detected for the first time in ticks. The tick species Rhipicephalus evertsi evertsi, Rhipicephalus (Boophilus) decoloratus, Hyalomma rufipes, Rhipicephalus appendiculatus, and Amblyomma hebraeum were associated with a broader pathogen range than previously known, and thus new vector-pathogen combinations are described. In addition, previously unknown coinfection patterns in tick salivary glands are reported.
\end{abstract}

(c) 2013 Elsevier GmbH. All rights reserved.

\section{Introduction}

Ticks are vectors of a great variety of tick-borne pathogens. In the recent decades, the development of molecular tools increased their known number and their known variety. The majority of tick-borne pathogen species that circulate between ticks and both game and livestock animals have been reported in the vertebrate hosts they infect and less in the ticks. Indeed, in early studies that aimed to detect tick-borne pathogens in wild African ungulates, animal sera were screened (Neitz and Du Toit, 1932; Neitz, 1935; Löhr and Meyer, 1973; Löhr et al., 1974; Carmichael and Hobday, 1975). More recently, in studies reporting tick-borne

\footnotetext{
* Corresponding author. Tel.: +41 32 7183043; fax: +41 327183001.

E-mail addresses: mirko.berggoetz@unine.ch (M. Berggoetz), melody.schmid@unine.ch (M. Schmid), daniel.ston@unine.ch (D. Ston), virginie.wyss@unine.ch (V. Wyss), Christine.chevillon@ird.fr (C. Chevillon), gnvramp@gmail.com (A.-M. Pretorius), lise.gern@unine.ch (L. Gern).
}

pathogens affecting game animals, host blood was examined, but not the associated tick species (Nijhof et al., 2003, 2005; Spitalska et al., 2005; Brothers et al., 2011; Oosthuizen et al., 2008, 2009; Pfitzer et al., 2011). In addition, several tick-borne pathogens with known tick vectors display a broader host range than formerly thought. An example is Theileria sp. (sable) that infects the sable (Hippotragus niger) and roan (H. equinus) antelope and that has been newly described in red hartebeest (Alcelaphus buselaphus) (Spitalska et al., 2005), cattle (Yusufmia et al., 2010), and nyala (Tragelaphus angassii) (Pfitzer et al., 2011). For these pathogens, a broader host range could mean that additional, so far unknown vectors, especially when associated with a wide host variety, might be involved in their transmission. From this point of view, the two-host tick $R$. e. evertsi, the three-host ticks Amblyomma hebraeum and $R$. appendiculatus as well as the one-host tick $R$. (Boophilus) decoloratus are good candidates. These tick species were recorded from a great variety of host species and are geographically widely distributed in southern Africa (Walker et al., 2000, 2003). 
Therefore, in this study, we focused on the presence of Babesia, Theileria, Anaplasma, and Ehrlichia in the salivary glands of different tick species collected from wild and domestic ruminants in South Africa. Detection of pathogens in salivary glands of ticks allowed us to distinguish pathogens that infected the ticks before they attached to the hosts from those taken up by ticks during their blood meal. Thus, this approach allows to evaluate the exposure of wild and domestic ruminants to tick-borne pathogens in their respective environments. The presence of a pathogen in tick salivary glands strongly suggests that it may be transmitted to the host, but this is not sufficient to prove its vector role, which would require transmission experiments. The purpose here was (i) to evaluate the possible role of ticks in the transmission of recently described pathogen species, (ii) to verify whether pathogens with a broad host range have a broader vector range than currently known, (iii) to investigate the exposure of game and livestock to tick-borne pathogens in their respective environments, and (iv) to obtain information on coinfections in ticks.

\section{Materials and methods}

\section{Study areas}

Ticks were collected in 2009 (May to July), 2010 (January to May), and 2011 (April to June) at 6 localities in 3 South African Provinces. In the Free State, 3 provincial nature reserves, TüssenDie-Riviere, Willem Pretorius, and Sandveld, as well as several livestock farms at their surroundings were investigated (highveld). In the Mpumalanga Province, one game farm and one livestock farm were investigated near Bethal (highveld). Finally, 5 game farms and 5 livestock farms were investigated in the Limpopo Province in the Thabazimbi and Lephalale areas (lowveld).

\section{Tick sampling}

At each locality, tick samplings were performed on domestic and wild animals within the same week. Ticks were sampled from an equal number of livestock animals living on farms sharing a common border with the reserves or game farms or located in the close surroundings (within the range of $40 \mathrm{~km}$ ). Ticks were collected from game animals during game capture, culling operations, and hunts. Cattle were maintained in holding facilities during sampling, and sheep were immobilised by hand in small camps. Hosts were visually examined for ticks; palpation helped to localise specimens attached on flanks, back, belly, neck, and legs. All the found ticks were removed with tweezers. Tick identification was performed according to Matthysse and Colbo (1987) and Walker et al. (2000, 2003). Ticks were pooled per species, host, and developmental stage and stocked in alcohol in 50-ml labelled tubes.

\section{Detection and identification of pathogen species in tick salivary} glands

From each individual host, a maximum of 10 males and 10 females from each tick species was analysed. To distinguish pathogens that had infected ticks before they attached to the host from those taken up by ticks during their current blood meal, each tick was dissected and the salivary glands of dissected ticks were analysed for pathogens. Salivary glands were carefully removed with tweezers - special attention was paid to avoid contamination with the midgut, and washed twice in PBS in 96-well plates (Milian ${ }^{\circledR}$, Geneva, Switzerland). Instruments were sterilised for a few seconds in $5 \mathrm{M} \mathrm{HCl}$ and $5 \mathrm{M} \mathrm{NaOH}$ (Aktas et al., 2009) and dried with sterile wipes between each dissection.

DNA from tick salivary glands was extracted using QIAamp ${ }^{\circledR}$ DNA Micro kit (Qiagen, Hombrechtikon, Switzerland) following the manufacturer's instructions with modifications. Tissue lysis buffer and proteinase $\mathrm{K}$ were added in the $1.5-\mathrm{ml}$ tubes before the salivary glands. DNA was stored at $-20^{\circ} \mathrm{C}$.

An approximately 500-base pair (bp) fragment of the $16 \mathrm{~S}$ ribosomal RNA (rRNA) gene spanning the hypervariable V1 region of the genera Anaplasma and Ehrlichia and an approximately 400-bp fragment of the 18S rRNA gene spanning the V4 hypervariable region of the genera Babesia and Theileria were amplified by PCR (Tonetti et al., 2009). Positive control, included in each run, consisted of DNA of A. phagocytophilum (provided by Ana Sofia Santos, Instituto Nacional de Saude, Lisboa, Portugal) and B. divergens (provided by Simona Casati, Institut Cantonal de Microbiologie, Bellinzone, Switzerland). PCR products were analysed using reverse line blotting (RLB) (Tonetti et al., 2009). In addition to the original 15 oligonucleotide probes, which are listed in Tonetti et al. (2009) (2 genus-specific Babesia/Theileria and Anaplasma/Ehrlichia, and 13 species-specific), 26 probes were added: one genus-specific Theileria spp. (Nagore et al., 2004) and 25 species-specific probes, B. ovis, B. crassa (Schnittger et al., 2004), B. major (Georges et al., 2001), Babesia sp. (sable) (Oosthuizen et al., 2008), B. caballi (Butler et al., 2008), B. occultans (Ros-Garcia et al., 2011), B. orientalis (Hea et al., 2011), B. gibsoni (from Pfitzer, 2009), B. rossi (Matjila et al., 2004), B. bicornis (Nijhof et al., 2003), B. motasi, Theileria sp. (greater kudu), Theileria sp.(sable)(Nijhof et al., 2005), T. separata, T. lestoquardi, T. ovis (Schnittger et al., 2004), T. buffeli (Gubbels et al., 1999), T. bicornis (Nijhof et al., 2003), Theileria sp. (buffalo) (Oura et al., 2004), T. equi (Butler et al., 2008), T. annulata (Georges et al., 2001), Ehrlichia sp. (Omatjenne) (Bekker et al., 2002), and 4 A. phagocytophilum (from Pfitzer, 2009) that replaced the original degenerated probe. Samples reacting only with the Babesia/Theileria probe were considered Babesia spp. since a genus-specific probe was included for the genus Theileria. To test for theoretical specificity, oligonucleotide probes were aligned with various sequences of targeted species available from the National Centre for Biotechnology Information (NCBI) using a software package: CLC Sequence Viewer 6 (CLC bio, Aarhus, Denmark).

\section{Sequencing}

PCR products that reacted only with genus-specific probes Babesia/Theileria, Theileria spp., or Anaplasma/Ehrlichia and did not hybridise with the panel of species-specific probes were sequenced. Prior sequencing, PCR products were purified using Wizard ${ }^{\circledR}$ SV and PCR Clean-Up System (Promega, Madison, USA) following the manufacturer's instructions except that we eluted with $35 \mu \mathrm{l}$ rather than $70 \mu \mathrm{l}$ Nuclease-Free Water. Sequencing was performed by Microsynth AG (Balgach, Switzerland). Sequences were compared and corrected with CLC Sequence Viewer 6 (CLC bio, Aarhus, Denmark) and Bioedit (Tom Hall Ibis Biosciences, Carlsbad). Corrected sequences were compared with available sequences retrieved from GenBank using NCBI Basic Local Alignment Search Tool (BLAST).

\section{Data analysis}

Data were analysed with "R" 2.14 for Windows (R Development Core Team, 2012. R: A language and environment for statistical computing. R Foundation for Statistical Computing, Vienna, Austria. ISBN 3-900051-07-0, URL: http://www.R-project.org/), using software packages (Skaug et al., 2010; Husson et al., 2012). A generalised linear mixed model (GLMM) with negative binominal errors was used to evaluate tick salivary gland infection (taking into account the 4 main infected tick species) collected from wild and domestic ruminants originating from 6 localities. In each locality, the significance of the factors LOCATION and HOST TYPE (i.e. ticks infesting wild vs. domestic ruminants) as well as the 
LOCATION-HOST TYPE interaction was assessed, $P$ values were considered significant when below 0.05 . In order to compute whether associations between pathogen species in coinfections were significant a permutation test proposed by Raup and Crick (1979), adapted to R by Clua et al. (2010) constrained to locations was used. Here, $P$ values were considered significant when below 0.0001 (Bonferroni corrected).

\section{Results}

\section{Tick sampling}

A total of 7364 ticks belonging to 13 species was collected from 13 ruminant species (Table 1 ). Five tick species contributed $96.5 \%$ of the total sampling: $R$. (B.) decoloratus $(n=2611), R$. e. evertsi $(n=2225)$, Margaropus winthemi $(n=1085)$, A. hebraeum $(n=767)$, and Hyalomma rufipes $(n=417)$, and $71 \%$ of them were collected from wild animals (Table 1 ). All tick species were detected in areas that were congruent with their known geographic distribution except Haemaphysalis silacea, which was recorded at Willem Pretorius game reserve (central Free State) (Supplementary Table S1). The 2 most abundant tick species were collected at each of the 6 sampling sites, $R$. (B.) decoloratus infested 9 ruminant species and $R$. e. evertsi all host species.

Supplementary material related to this article can be found, in the online version, at doi:10.1016/j.ttbdis.2013.10.003.

\section{Infection of tick salivary glands}

Among the 2117 examined ticks, 329 (15.5\%) were infected, and they harboured 397 infections (Table 2). The majority (95\%) of the infections were observed in 4 tick species: $R$. e. evertsi harboured $48.6 \%$ of the infections (193/397), R. (B.) decoloratus $24.7 \%$ (98/397), A. hebraeum $11.8 \%$ (47/397), and Hy. rufipes 9.6\% (38/397) (Table 2). Five tick species (Ixodes rubicundus, M. winthemi, Hae. silacea, Otobius megnini, and $R$. zambeziensis) were free of pathogens. One hundred sixty-eight infections were identified at genus level only: 69 Babesia spp., 56 Theileria spp., and 43 Anaplasma or Ehrlichia spp. Sequencing of all these samples did not allow further identification. The remaining infections $(n=229)(57.7 \%)$ were identified to species level and were assigned to 23 pathogen species, all known to occur in South Africa, except T. annulata (Table 2). Among them were 46 identified by sequencing (Table 3 ). Theileria sp. (sable) ( $n=38 ; 9.6 \%)$, . buffeli $(n=37 ; 9.3 \%)$, T. bicornis $(n=27 ; 6.8 \%)$, Ehrlichia sp. (EU191229.1) $(n=23 ; 5.8 \%)$, and T. separata $(n=18$; $4.5 \%$ ) were the most frequent (Table 2 ).

\section{Pathogen distribution among tick species}

Nine pathogen species were detected in one tick species only. Theileria equi, T. separata, A. ovis, and A. platys were detected in $R$. e. evertsi; B. occultans and Babesia sp. (sable) were only detected in $H y$. rufipes, and Theileria sp. (kudu), Anaplasma centrale, and Ehrlichia ruminantium in $R$. appendiculatus, $R$. gertrudae, and A. hebraeum, respectively (Table 2 ). Seven pathogens were detected in 2 tick species. Babesia bigemina, B. caballi, A. bovis, T. ovis, and Theileria sp. (giraffe) were detected in $R$. (B.) decoloratus and $R$. e. evertsi. Theileria bicornis was detected in $R$. e. evertsi and $R$. appendiculatus. Finally, $T$. mutans was detected in $R$. e. evertsi and A. hebraeum. Four pathogen species were identified in 3 tick species. Theileria sp. (sable) was detected in $R$. e. evertsi, $R$. (B.) decoloratus, and A. hebraeum. Theileria taurotragi was detected in $R$. appendiculatus, $R$. e. evertsi, and Hy. rufipes. Anaplasma marginale was detected in $R$. gertrudae, $R$. (B.) decoloratus, and R. e. evertsi.Ehrlichia sp.(EU191229.1) was detected in R. (B.) decoloratus, R. e. evertsi, and Hy. rufipes. Finally, T. buffeli and $E$. ovina were detected in 4 tick species: Theileria buffeli was detected in R. (B.) decoloratus, R. e. evertsi, Hy. rufipes, and R. appendiculatus, while E. ovina was detected in $R$. (B.) decoloratus, $R$. e. evertsi, Hy. rufipes, and R. gertrudae.

\section{Salivary gland infection of ticks attached to wild versus domestic ruminants}

Ticks that were attached to wild ruminants showed an infection prevalence of 23.3\% (227/973) and harboured 284 infections, while ticks attached to domestic ruminants displayed an infection prevalence of $11.5 \%$ (102/885) and harboured 113 infections (Tables $4 \mathrm{a}$ and $4 \mathrm{~b})$. The differences were mainly due to Theileria spp. (16.1\% vs. $8.4 \%$ ), Theileria sp. (sable) (12.6\% vs. $1.7 \%)$, T. buffeli (10.2\% vs. $6.7 \%)$, T. bicornis ( $7.4 \%$ vs. $5 \%$ ), and T. separata (4.9\% vs. $3.4 \%$ ) (Tables $4 \mathrm{a}$ and $4 \mathrm{~b}$ ).

Taking into account the 4 main infected tick species [R. (B.) decoloratus, $R$. e. evertsi, A. hebraeum, and Hy. rufipes], salivary gland infection (prevalence of infection and mean density of pathogens) varied significantly among the sampling localities (Table 5). The infection pattern was impacted by the factors LOCATION and HOST TYPE as well as by the interaction of both factors (Glmm analyses $P<0.001$ for the factors LOCATION, HOST TYPE, and their interactions). In contrast,the intensities of infection did not significantly differ among host types and/or locations at the 5\% risk (data not shown). In Sandveld, Thabazimbi, and Lephalale, infection prevalence and mean density of pathogens were significantly higher in salivary glands of ticks infesting wild than domestic hosts. Similarly, the tick mean density was significantly higher on wild animals than on domestic animals in Sandveld and Thabazimbi (data not shown).

\section{Coinfections}

Among infections identified at species level, 93 (44.7\%) were coinfections: 57 (61.3\%) involved 2 pathogens, 24 (25.8\%) 3, and $12(12.9 \%) 4$ (data not shown). Rhipicephalus e. evertsi showed the highest prevalence of coinfections ( $n=67 ; 72 \%$ ), followed by $R$. (B.) decoloratus $(n=21 ; 22.6 \%), R$. appendiculatus $(n=3 ; 3.2 \%)$, and $H y$. rufipes ( $n=2 ; 2.2 \%$ ) (data not shown). Six associations involving 5 pathogen species were significant: Theileria sp. (sable) with $T$. separata; Theileria sp. (sable) with $T$. bicornis; $T$. separata with $T$. bicornis; T. separata with T. buffeli; T. bicornis with T. buffeli, and $T$. buffeli with $B$. caballi $[P<0.0004$, permutation test constrained to locations (Raup and Crick, 1979, modified by Clua et al., 2010)] (Supplementary Table S2).

Supplementary material related to this article can be found, in the online version, at doi:10.1016/j.ttbdis.2013.10.003.

\section{Discussion}

In this study, 13 tick species collected from wild and domestic ruminants were analysed by RLB hybridisation using probes allowing identification of tick-borne pathogens at the genus and species level. The majority $(n=229)$ of the 397 infections were identified at species level. Among those, 46 infections assigned to 7 species were identified by sequencing: Ehrlichia sp. (EU191229.1), E. ovina, Theileria sp. (giraffe), A. platys, A. ovis, A. marginale, and A. bovis. No species-specific probes were included in the assay for the former 4 species. The latter 3 species did not react with their specific oligonucleotide probes. For A. ovis and A. marginale, this was probably due to the forward position of the probes on the amplified sequence, while for A. bovis, it was probably due to one base pair difference in the amplified sequences. The remaining 168 infections were only identified at the genus level, even after sequencing, probably because of the presence of multiple pathogens (Microsynth, pers. communication) for which no probes were available. 
Table 1

Host infestation $(n=128)$ by 13 tick species in six localities in South Africa.

\begin{tabular}{|c|c|c|c|c|c|c|}
\hline \multirow[t]{2}{*}{ Tick (bold) and host species } & \multirow{2}{*}{$\begin{array}{l}\text { Hosts } \\
\text { No. infested/examined }\end{array}$} & \multicolumn{5}{|c|}{ Number of ticks } \\
\hline & & Larvae & Nymphs & Males & Females & Total \\
\hline \multicolumn{7}{|l|}{ Rhipicephalus (B.) decoloratus } \\
\hline Common eland (Tragelaphus oryx) & $1 / 9(11 \%)$ & 9 & 91 & 135 & 97 & 332 \\
\hline Greater kudu (T. strepsiceros) & $3 / 8(38 \%)$ & 1 & 59 & 35 & 35 & 130 \\
\hline Impala (Aepyceros melampus) & $2 / 7(29 \%)$ & 213 & 483 & 434 & 207 & 1337 \\
\hline Southern giraffe (Giraffa camelopardalis) & $5 / 5(100 \%)$ & 0 & 0 & 6 & 23 & 29 \\
\hline Blue wildebeest (Connochaetes taurinus) & $4 / 5(80 \%)$ & 8 & 10 & 40 & 53 & 111 \\
\hline Sable antelope (Hippotragus niger) & $2 / 2(100 \%)$ & 0 & 14 & 37 & 45 & 96 \\
\hline Gemsbok (Oryx gazella gazella) & $1 / 1(100 \%)$ & 33 & 52 & 72 & 56 & 213 \\
\hline Cattle (Bos primigenius taurus/B. p. taurus/indicus) & $17 / 50(34 \%)$ & 0 & 21 & 136 & 204 & 361 \\
\hline Sheep (Ovis aries) & $2 / 14(14 \%)$ & 0 & 0 & 0 & 2 & 2 \\
\hline Total & $37 / 101(37 \%)$ & 264 & 730 & 895 & 722 & 2611 \\
\hline \multicolumn{7}{|l|}{ R. e. evertsi } \\
\hline African buffalo (Syncerus caffer) & $14 / 15(93 \%)$ & 0 & 0 & 330 & 112 & 442 \\
\hline Common eland ( $T$. oryx $)$ & $9 / 9(100 \%)$ & 0 & 2 & 262 & 27 & 291 \\
\hline Greater kudu (T. strepsiceros) & $4 / 8(50 \%)$ & 7 & 24 & 7 & 0 & 38 \\
\hline Impala (A. melampus) & $3 / 7(43 \%)$ & 0 & 49 & 11 & 2 & 62 \\
\hline Blue wildebeest (C. taurinus) & $5 / 5(100 \%)$ & 0 & 0 & 48 & 38 & 86 \\
\hline Springbok (Antidorcas marsupialis) & $4 / 6(67 \%)$ & 0 & 0 & 6 & 5 & 11 \\
\hline Southern giraffe (G. camelopardalis) & $4 / 5(80 \%)$ & 0 & 0 & 15 & 12 & 27 \\
\hline Blesbok (Damaliscus pygarus phillipsi) & $1 / 3(33 \%)$ & 3 & 5 & 0 & 0 & 8 \\
\hline Black wildebeest (C. gnou) & $2 / 3(67 \%)$ & 0 & 0 & 3 & 3 & 6 \\
\hline Sable antelope (H. niger) & $2 / 2(100 \%)$ & 0 & 0 & 9 & 1 & 10 \\
\hline Gemsbok (O.g. gazella) & $1 / 1(100 \%)$ & 0 & 0 & 26 & 5 & 31 \\
\hline Cattle (B.p.taurus/B.p.t./indicus) & $49 / 50(98 \%)$ & 189 & 424 & 431 & 153 & 1197 \\
\hline Sheep (O. aries) & $5 / 14(36 \%)$ & 0 & 0 & 14 & 2 & 16 \\
\hline Total & $101 / 128(79 \%)$ & 199 & 504 & 1162 & 360 & 2225 \\
\hline \multicolumn{7}{|l|}{ Margaropus winthemi } \\
\hline Common eland (T. oryx) & $6 / 9(67 \%)$ & 11 & 505 & 287 & 234 & 1037 \\
\hline Greater kudu (T. strepsiceros) & $1 / 8(13 \%)$ & 0 & 0 & 0 & 9 & 9 \\
\hline Gemsbok (O.g. gazella) & $1 / 1(100 \%)$ & 1 & 0 & 0 & 27 & 28 \\
\hline Cattle (B. p. taurus/B. p.t./indicus) & $3 / 50(6 \%)$ & 0 & 0 & 0 & 11 & 11 \\
\hline Total & $11 / 68(16 \%)$ & 12 & 505 & 287 & 281 & 1085 \\
\hline \multicolumn{7}{|l|}{ Amblyomma hebraeum } \\
\hline Southern giraffe (G. camelopardalis) & $5 / 5(100 \%)$ & 0 & 0 & 307 & 117 & 424 \\
\hline Cattle (B. p.taurus/B. p.t./indicus) & $10 / 50(20 \%)$ & 0 & 0 & 229 & 114 & 343 \\
\hline Total & $15 / 55(27 \%)$ & 0 & 0 & 536 & 231 & 767 \\
\hline Hyalomma m. rufipes & & & & & & \\
\hline Buffalo (S. caffer) & $8 / 15(53 \%)$ & 0 & 0 & 13 & 8 & 21 \\
\hline Common eland ( $T$. oryx) & $9 / 9(100 \%)$ & 0 & 0 & 215 & 14 & 229 \\
\hline Southern giraffe (G. camelopardalis) & $4 / 5(80 \%)$ & 0 & 0 & 19 & 5 & 24 \\
\hline Blue wildebeest (C. taurinus) & $3 / 5(60 \%)$ & 0 & 0 & 4 & 7 & 11 \\
\hline Sable antelope (H. niger) & $1 / 2(50 \%)$ & 0 & 0 & 1 & 0 & 1 \\
\hline Cattle (B.p.taurus/B.p.t./indicus) & $28 / 50(56 \%)$ & 0 & 0 & 80 & 49 & 129 \\
\hline Sheep (O. aries) & $2 / 14(14 \%)$ & 0 & 0 & 1 & 1 & 2 \\
\hline Total & $55 / 100(55 \%)$ & 0 & 0 & 333 & 84 & 417 \\
\hline Ixodes rubicundus & & & & & & \\
\hline Common eland ( $T$. oryx) & $4 / 9(44 \%)$ & 0 & 0 & 22 & 32 & 54 \\
\hline Greater kudu (T. strepsiceros) & $5 / 8(63 \%)$ & 0 & 0 & 16 & 34 & 50 \\
\hline Springbok (A. marsupialis) & $5 / 6(83 \%)$ & 0 & 0 & 9 & 11 & 20 \\
\hline Cattle (B.p.taurus/B.p.t./indicus) & $4 / 50(8 \%)$ & 0 & 0 & 7 & 21 & 28 \\
\hline Sheep (O. aries) & $2 / 14(14 \%)$ & 0 & 0 & 1 & 1 & 2 \\
\hline Total & $20 / 87(23 \%)$ & 0 & 0 & 55 & 99 & 154 \\
\hline R. appendiculatus & & & & & & \\
\hline Impala (A. melampus) & $1 / 7(14 \%)$ & 0 & 0 & 4 & 0 & 4 \\
\hline Southern giraffe (G. camelopardalis) & $1 / 5(20 \%)$ & 0 & 0 & 9 & 4 & 13 \\
\hline Cattle (B. p. taurus/B. p.t./indicus) & $3 / 50(6 \%)$ & 0 & 0 & 19 & 2 & 21 \\
\hline Total & $5 / 62(8 \%)$ & 0 & 0 & 32 & 6 & 38 \\
\hline R. gertrudae & & & & & & \\
\hline African buffalo (S. caffer) & $1 / 15(7 \%)$ & 0 & 0 & 21 & 1 & 22 \\
\hline Common eland (T. oryx) & $1 / 9(11 \%)$ & 0 & 0 & 8 & 0 & 8 \\
\hline Total & $2 / 24(8 \%)$ & 0 & 0 & 29 & 1 & 30 \\
\hline R. warburtoni & & & & & & \\
\hline African Buffalo (S. caffer) & $1 / 15(7 \%)$ & 0 & 0 & 1 & 0 & 1 \\
\hline Common eland (T. oryx) & $2 / 9(22 \%)$ & 0 & 0 & 13 & 1 & 14 \\
\hline Total & $3 / 24(12 \%)$ & 0 & 0 & 14 & 1 & 15 \\
\hline Haemaphysalis silacea & & & & & & \\
\hline African buffalo (S. caffer) & $2 / 15(13 \%)$ & 0 & 0 & 0 & 8 & 8 \\
\hline Total & $2 / 15(13 \%)$ & 0 & 0 & 0 & 8 & 8 \\
\hline R. (B.) microplus & & & & & & \\
\hline Cattle (B. p.taurus/B. p.t./indicus) & $1 / 50(2 \%)$ & 0 & 3 & 1 & 3 & 7 \\
\hline Otobius megnini & & & & & & \\
\hline Cattle (B.p.taurus/B. p.t./indicus) & $2 / 50(4 \%)$ & 0 & 5 & 0 & 0 & 5 \\
\hline Total & $2 / 50(4 \%)$ & 0 & 5 & 0 & 0 & 5 \\
\hline R. zambeziensis & & & & & & \\
\hline Impala (A. melampus) & $1 / 7(14 \%)$ & 0 & 0 & 2 & 0 & 2 \\
\hline Total & - & 475 & 1747 & 3346 & 1796 & 7364 \\
\hline
\end{tabular}


Table 2

Infection of 8 out of 13 tick species by 23 pathogen species collected from 64 wild and 64 domestic ruminants in South Africa.

\begin{tabular}{|c|c|c|c|c|c|c|c|c|c|c|c|c|c|c|}
\hline Pathogens/ticks & $\begin{array}{l}\text { R. (B.) } \\
\text { decoloratus }\end{array}$ & $\begin{array}{l}\text { R.e. } \\
\text { evertsi }\end{array}$ & $\begin{array}{l}\text { A. } \\
\text { hebraeum }\end{array}$ & $\begin{array}{l}\text { H. } m . \\
\text { rufipes }\end{array}$ & $\begin{array}{l}R . \\
\text { appendiculatus }\end{array}$ & $\begin{array}{l}R . \\
\text { gertrudae }\end{array}$ & $\begin{array}{l}R .(B .) \\
\text { microplus }\end{array}$ & $\begin{array}{l}R . \\
\text { warburtoni }\end{array}$ & $\begin{array}{l}\text { I. } \\
\text { rubicundus }\end{array}$ & $\begin{array}{l}\text { M. } \\
\text { winthemi }\end{array}$ & $\begin{array}{l}\mathrm{H} . \\
\text { silacea }\end{array}$ & $\begin{array}{l}\text { O. } \\
\text { megnini }\end{array}$ & $\begin{array}{l}R . \\
\text { zambeziensis }\end{array}$ & $\begin{array}{l}\text { \% of pathogen } \\
\text { species }^{\mathrm{D}}\end{array}$ \\
\hline $\mathrm{B} / \mathrm{T}_{\text {genus }}{ }^{\mathrm{g}}$ & 24 & 30 & 6 & 6 & 3 & 0 & 0 & 0 & 0 & 0 & 0 & 0 & 0 & 69 (17.4\%) \\
\hline B. occultans & 0 & 0 & 0 & $2^{\mathrm{a}, \mathrm{f}}$ & 0 & 0 & 0 & 0 & 0 & 0 & 0 & 0 & 0 & $2(0.5 \%)$ \\
\hline B. bigemina & $5^{b, c, e, f}$ & $1^{\mathrm{f}}$ & 0 & 0 & 0 & 0 & 0 & 0 & 0 & 0 & 0 & 0 & 0 & $6(1.5 \%)$ \\
\hline B. caballi & $7^{b, c, e}$ & $5^{b, c}$ & 0 & 0 & 0 & 0 & 0 & 0 & 0 & 0 & 0 & 0 & 0 & $12(3 \%)$ \\
\hline B. sp. Sable & 0 & 0 & 0 & $1^{\mathrm{b}}$ & 0 & 0 & 0 & 0 & 0 & 0 & 0 & 0 & 0 & $1(0.3 \%)$ \\
\hline T. spp. & 15 & 23 & 13 & 2 & 3 & 0 & 0 & 0 & 0 & 0 & 0 & 0 & 0 & $56(14.1 \%)$ \\
\hline T. bicornis & 0 & $\mathbf{2 6}^{\mathrm{a}, \mathrm{b}, \mathrm{b}, \mathrm{e}}$ & 0 & 0 & $\mathbf{1}^{\mathrm{e}}$ & 0 & 0 & 0 & 0 & 0 & 0 & 0 & 0 & $27(6.8 \%)$ \\
\hline T. ovis & $2^{\mathrm{e}}$ & $2^{\mathrm{a}, \mathrm{c}}$ & 0 & 0 & 0 & 0 & 0 & 0 & 0 & 0 & 0 & 0 & 0 & $4(1 \%)$ \\
\hline T. equi & 0 & $14^{\mathrm{a}, \mathrm{a}, \mathrm{c}, \mathrm{e}, \mathrm{f}}$ & 0 & 0 & 0 & 0 & 0 & 0 & 0 & 0 & 0 & 0 & 0 & $14(3.5 \%)$ \\
\hline T. separata & 0 & $18^{\mathrm{b}, \mathrm{c}, \mathrm{d}, \mathrm{e}}$ & 0 & 0 & 0 & 0 & 0 & 0 & 0 & 0 & 0 & 0 & 0 & $18(4.5 \%)$ \\
\hline T. sp. (sable) & $\mathbf{9}^{\mathrm{c}, \mathrm{e}, \mathrm{f}}$ & $24^{\mathrm{b}, \mathrm{c}, \mathrm{d}, \mathrm{e}}$ & $\mathbf{5}^{\text {eff }}$ & 0 & 0 & 0 & 0 & 0 & 0 & 0 & 0 & 0 & 0 & 38 (9.6\%) \\
\hline T. buffeli & $17^{b, c, e, f}$ & $17^{\mathrm{b}, \mathrm{c}, \mathrm{e}, \mathrm{f}}$ & 0 & $2^{\mathrm{a}, \mathrm{f}}$ & $\mathbf{1}^{\mathrm{e}}$ & 0 & 0 & 0 & 0 & 0 & 0 & 0 & 0 & 37 (9.3\%) \\
\hline T. taurotragi & 0 & $1^{\mathrm{f}}$ & 0 & $\mathbf{1}^{\mathrm{b}}$ & $2^{e}$ & 0 & 0 & 0 & 0 & 0 & 0 & 0 & 0 & $4(1 \%)$ \\
\hline T. annulata & $4^{\mathrm{b}, \mathrm{e}}$ & $2^{b, e}$ & 0 & 0 & 0 & 0 & 0 & 0 & 0 & 0 & 0 & 0 & 0 & $6(1.5 \%)$ \\
\hline T. sp. (kudu) & 0 & 0 & 0 & 0 & $\mathbf{1}^{\mathrm{e}}$ & 0 & 0 & 0 & 0 & 0 & 0 & 0 & 0 & $1(0.3 \%)$ \\
\hline T. mutans & 0 & $\mathbf{1}^{\mathrm{f}}$ & $1^{\mathrm{f}}$ & 0 & 0 & 0 & 0 & 0 & 0 & 0 & 0 & 0 & 0 & $2(0.5 \%)$ \\
\hline T. sp. (giraffe) & $\mathbf{1}^{\mathrm{f}}$ & $\mathbf{1}^{\mathrm{f}}$ & 0 & 0 & 0 & 0 & 0 & 0 & 0 & 0 & 0 & 0 & 0 & $2(0.5 \%)$ \\
\hline A/E genus & 3 & 7 & 18 & 11 & 0 & 0 & 1 & 3 & 0 & 0 & 0 & 0 & 0 & $43(10.8 \%)$ \\
\hline A. bovis & $\mathbf{1}^{\mathrm{b}}$ & $11^{a, b, c}$ & 0 & 0 & 0 & 0 & 0 & 0 & 0 & 0 & 0 & 0 & 0 & $12(3 \%)$ \\
\hline A. centrale & 0 & 0 & 0 & 0 & 0 & $\mathbf{1}^{\mathrm{a}}$ & 0 & 0 & 0 & 0 & 0 & 0 & 0 & $1(0.3 \%)$ \\
\hline A. marginale & $1^{\mathrm{d}}$ & $1^{\mathrm{c}}$ & 0 & 0 & 0 & $4^{\mathrm{a}}$ & 0 & 0 & 0 & 0 & 0 & 0 & 0 & $6(1.5 \%)$ \\
\hline A. ovis & 0 & $3^{c, f}$ & 0 & 0 & 0 & 0 & 0 & 0 & 0 & 0 & 0 & 0 & 0 & $3(0.8 \%)$ \\
\hline E. sp. (EU191229.1) & $8^{\mathrm{b}, \mathrm{e}}$ & $3^{\mathrm{c}, \mathrm{f}}$ & 0 & $12^{\mathrm{a}, \mathrm{c}}$ & 0 & 0 & 0 & 0 & 0 & 0 & 0 & 0 & 0 & $23(5.8 \%)$ \\
\hline E. ovina & $\mathbf{1}^{\mathrm{c}}$ & $2^{\mathrm{a}, \mathrm{c}}$ & 0 & $\mathbf{1}^{\mathrm{a}}$ & 0 & $\mathbf{1}^{\mathrm{a}}$ & 0 & 0 & 0 & 0 & 0 & 0 & 0 & $5(1.3 \%)$ \\
\hline E. ruminantium & 0 & 0 & $4^{\mathrm{f}}$ & 0 & 0 & 0 & 0 & 0 & 0 & 0 & 0 & 0 & 0 & $4(1 \%)$ \\
\hline A. platys & 0 & $1^{\mathrm{a}}$ & 0 & 0 & 0 & 0 & 0 & 0 & 0 & 0 & 0 & 0 & 0 & $1(0.3 \%)$ \\
\hline $\begin{array}{l}\text { Prevalence of } \\
\text { infection }^{A}\end{array}$ & $\begin{array}{l}84 / 395 \\
(21.3 \%)\end{array}$ & $\begin{array}{l}147 / 909 \\
(16.2 \%)\end{array}$ & $\begin{array}{l}42 / 233 \\
(18 \%)\end{array}$ & $\begin{array}{l}37 / 259 \\
(14.3 \%)\end{array}$ & $9 / 26(34.6 \%)$ & $\begin{array}{l}6 / 18 \\
(33.3 \%)\end{array}$ & $1 / 4(25 \%)$ & $\begin{array}{l}3 / 14 \\
(21.4 \%)\end{array}$ & $0 / 131$ & $0 / 116$ & $0 / 7$ & $0 / 4$ & $0 / 1$ & $\begin{array}{l}329 / 2117 \\
(15.5 \%)\end{array}$ \\
\hline $\begin{array}{l}\text { Pathogen mean } \\
\text { density }^{\mathrm{B}}\end{array}$ & $98 / 395(0.25)$ & $\begin{array}{l}193 / 909 \\
(0.21)\end{array}$ & $\begin{array}{l}47 / 233 \\
(0.20)\end{array}$ & $\begin{array}{l}38 / 259 \\
(0.15)\end{array}$ & $11 / 26(0.42)$ & $\begin{array}{l}6 / 18 \\
(0.33)\end{array}$ & $1 / 4(0.25)$ & $3 / 14(0.21)$ & $0 / 131$ & $0 / 116$ & $0 / 7$ & $0 / 4$ & $0 / 1$ & $\begin{array}{l}397 / 2117 \\
(0.19)\end{array}$ \\
\hline $\begin{array}{l}\text { Intensity of } \\
\text { infection }^{C}\end{array}$ & $98 / 84(1.17)$ & $\begin{array}{l}193 / 147 \\
(1.31)\end{array}$ & $\begin{array}{l}47 / 42 \\
(1.12)\end{array}$ & $\begin{array}{l}38 / 37 \\
(1.03)\end{array}$ & $11 / 9(1.22)$ & $6 / 6(1)$ & $1 / 1(1)$ & $3 / 3(1)$ & 0 & 0 & 0 & 0 & 0 & $397 / 329(1.21)$ \\
\hline
\end{tabular}

A No. of infected ticks (data not shown)/no. of tested ticks.

${ }^{\text {B }}$ No. of infections/no. of tested ticks.

C No. of infections/no. of infected ticks.

D \% of the given pathogen species among the 397 infections.

Locations of the infections identified at species level: a, Tüssen-Die-Riviere; b, Willem Pretorius; c, Sandveld; d, Bethal; e, Thabazimbi; f, Lephalale.

In bold: new vector-pathogen combinations.

${ }^{g} B / T$ : includes infections reacting with this probe only; considered as belonging to the genus Babesia. 
Table 3

Pathogen species identified from tick salivary glands by sequencing.

\begin{tabular}{|c|c|c|c|c|}
\hline Identified sequence & Homology with & $\%$ of homology & Species name & Identified from \\
\hline KF414714 & EU191229.1 & $100 \%$ & Unnamed (E.sp.) & H. m. rufipes $(n=12)$ \\
\hline KF414714 & EU191229.1 & $100 \%$ & Unnamed (E. sp.) & R. (B.) decoloratus $(n=8)$ \\
\hline KF414714 & EU191229.1 & $100 \%$ & Unnamed (E. sp.) & R. e. evertsi $(n=3)$ \\
\hline KF414715 & AF318946.1 & $99 \%$ & E. ovina & R. e. evertsi $(n=2)$ \\
\hline KF414715 & AF318946.1 & $99 \%$ & E. ovina & H.m.rufipes $(n=1)$ \\
\hline KF414715 & AF318946.1 & $99 \%$ & E. ovina & R. gertrudae $(n=1)$ \\
\hline KF414715 & AF318946.1 & $99 \%$ & E. ovina & R. (B.) decoloratus $(n=1)$ \\
\hline KF414716 & FJ155997.1 & $100 \%$ & A. bovis & R. e. evertsi $(n=11)$ \\
\hline KF414717 & EU191231.1 & $100 \%$ & A. ovis & R. e. evertsi $(n=3)$ \\
\hline KF414718 & FJ155998.1 & $99 \%$ & A. marginale & R. gertrudae $(n=1)$ \\
\hline KF414719 & AY040853.1 & $99 \%$ & A. platys & R. e. evertsi $(n=1)$ \\
\hline KF414720 & FJ213583.1 & $100 \%$ & T. sp. (giraffe) & R. e. evertsi $(n=1)$ \\
\hline KF414720 & FJ213583.1 & $100 \%$ & T. sp. (giraffe) & R. (B.) decoloratus $(n=1)$ \\
\hline
\end{tabular}

The geographic distribution of all tick and pathogen species was congruent with their known geographic distribution except for Hae. silacea, T. annulata, and Ehrlichia sp. (EU191229.1). The tick Hae. silacea was collected at Willem Pretorius game reserve in the centre of the Free State. Its known geographic distribution is the north-eastern KwaZulu-Natal and the Eastern Cape Province (Norval, 1975; Walker, 1991; Horak et al., 1991, 2007). In Africa, T. annulata occurs only in northern Africa (Pipano, 1994; Jacquiet et al., 1990). Here, T. annulata was detected in the salivary glands of $4 R$. (B.) decoloratus and $2 R$. e. evertsi at Thabazimbi and in the Free State (Willem Pretorius) where they were sharing the same individual hosts. All T. annulata infections were identified through hybridisation with RLB-probes designed by Georges et al. (2001). Cross reactivity with pathogens identified in this study can be excluded. Whether cross reactivity occurred with an alternative or unknown Theileria species remains a possibility. Unfortunately, sequencing of these samples was not successful. Thus, the finding of this economically important cattle parasite in South Africa must be considered with caution. This pathogen is not discussed further, and Ehrlichia sp. (EU191229.1) will be discussed elsewhere (Berggoetz et al., unpublished).

Among the observed tick-pathogen combinations, 17 were congruent with the literature. Among those, 10 were recorded in the salivary glands of $R$. e. evertsi: B. bigemina (Büscher, 1988), B. caballi (De Waal and van Heerden, 1994), T. ovis (Jansen and Neitz, 1956), T. equi (De Waal and Potgieter, 1987), T. separata (Jansen and Neitz, 1956), Theileria sp. (sable) (Steyl et al., 2012), T. taurotragi (Lawrence et al., 1994a), A. marginale (Potgieter, 1981), A. ovis (Kaufmann, 1996), and E. ovina (Neitz, 1956). The remaining 7 known vector-pathogen combinations were recorded in the salivary glands of 4 tick species: $B$. bigemina (Potgieter and Els, 1977), T. ovis (Jansen and Neitz, 1956), and A. marginale (Potgieter, 1981) in R. (B.) decoloratus; T. mutans (Lawrence et al., 1994a) and E. ruminantium (Bezuidenhout et al., 1994) in A. hebraeum; B. occultans

Table 4a

Pathogens identified in seven tick species collected from 64 wild ruminants.

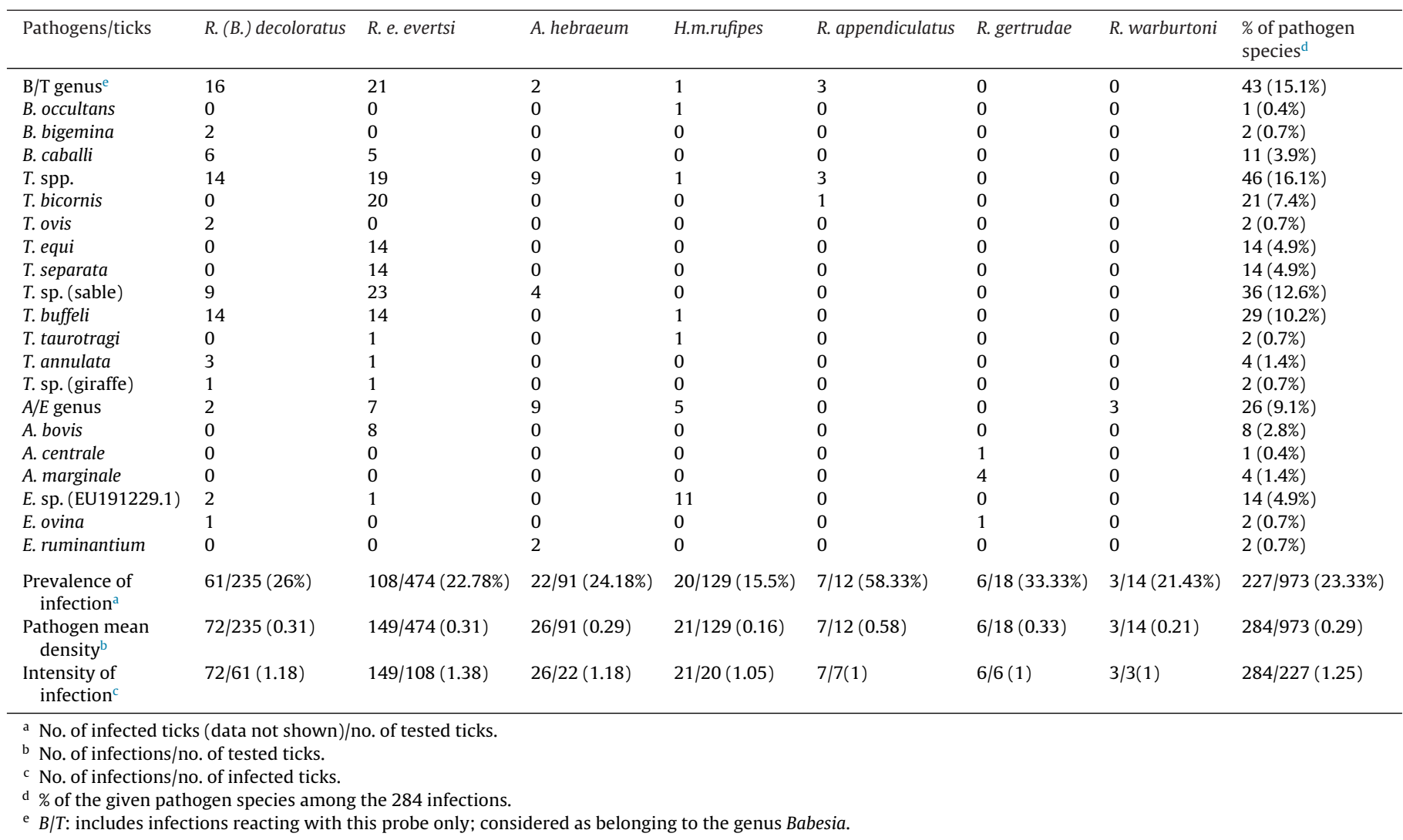


Table 4b

Pathogens identified in six tick species collected from 64 domestic ruminants.

\begin{tabular}{|c|c|c|c|c|c|c|c|}
\hline Pathogens/ticks & R. (B.) decoloratus & R. e. evertsi & A. hebraeum & H. m. rufipes & R. appendiculatus & R. (B.) microplus & $\begin{array}{l}\% \text { of pathogen } \\
\text { species }^{\mathrm{d}}\end{array}$ \\
\hline $\mathrm{B} / \mathrm{T}_{\text {genus }}{ }^{\mathrm{e}}$ & 8 & 9 & 4 & 5 & 0 & 0 & $26(21.8 \%)$ \\
\hline B. occultans & 0 & 0 & 0 & 1 & 0 & 0 & $1(0.8 \%)$ \\
\hline B. bigemina & 3 & 1 & 0 & 0 & 0 & 0 & $4(3.4 \%)$ \\
\hline B. caballi & 1 & 0 & 0 & 0 & 0 & 0 & $1(0.8 \%)$ \\
\hline B. sp. (sable) & 0 & 0 & 0 & 1 & 0 & 0 & $1(0.8 \%)$ \\
\hline T. spp. & 1 & 4 & 4 & 1 & 0 & 0 & $10(8.4 \%)$ \\
\hline T. bicornis & 0 & 6 & 0 & 0 & 0 & 0 & $6(5 \%)$ \\
\hline T. ovis & 0 & 2 & 0 & 0 & 0 & 0 & $2(1.7 \%)$ \\
\hline T. separata & 0 & 4 & 0 & 0 & 0 & 0 & $4(3.4 \%)$ \\
\hline T. sp. (sable) & 0 & 1 & 1 & 0 & 0 & 0 & $2(1.7 \%)$ \\
\hline T. buffeli & 3 & 3 & 0 & 1 & 1 & 0 & $8(6.7 \%)$ \\
\hline T. taurotragi & 0 & 0 & 0 & 0 & 2 & 0 & $2(1.7 \%)$ \\
\hline T. annulata & 1 & 1 & 0 & 0 & 0 & 0 & $2(1.7 \%)$ \\
\hline T. sp. (kudu) & 0 & 0 & 0 & 0 & 1 & 0 & $1(0.8 \%)$ \\
\hline T. mutans & 0 & 1 & 1 & 0 & 0 & 0 & $2(1.7 \%)$ \\
\hline A/E genus & 1 & 0 & 9 & 6 & 0 & 1 & $17(14.3 \%)$ \\
\hline A. bovis & 1 & 3 & 0 & 0 & 0 & 0 & $4(3.4 \%)$ \\
\hline A. marginale & 1 & 1 & 0 & 0 & 0 & 0 & $2(1.7 \%)$ \\
\hline A. ovis & 0 & 3 & 0 & 0 & 0 & 0 & $3(2.5 \%)$ \\
\hline E. sp. (EU191229.1) & 6 & 2 & 0 & 1 & 0 & 0 & $9(7.6 \%)$ \\
\hline E. ovina & 0 & 2 & 0 & 1 & 0 & 0 & $3(2.5 \%)$ \\
\hline E. ruminantium & 0 & 0 & 2 & 0 & 0 & 0 & $2(1.7 \%)$ \\
\hline A. platys & 0 & 1 & 0 & 0 & 0 & 0 & $1(0.8 \%)$ \\
\hline Prevalence of infection ${ }^{\mathrm{a}}$ & $23 / 160(14.4 \%)$ & $39 / 435(9 \%)$ & $20 / 142(14.1 \%)$ & $17 / 130(13.1 \%)$ & $2 / 14(14.3 \%)$ & $1 / 4(25 \%)$ & $102 / 885(11.5 \%)$ \\
\hline Pathogen mean density ${ }^{\mathrm{b}}$ & $26 / 160(0.16)$ & $44 / 435(0.10)$ & $21 / 142(0.15)$ & $17 / 130(0.13)$ & $4 / 14(0.29)$ & $1 / 4(0.25)$ & $113 / 885(0.13)$ \\
\hline Intensity of infection ${ }^{c}$ & $26 / 23(1.13)$ & $44 / 39(1.13)$ & $21 / 20(1.05)$ & $17 / 17(1)$ & $4 / 2(2)$ & $1 / 1(1)$ & $113 / 102(1.11)$ \\
\hline
\end{tabular}

a No. of infected ticks (data not shown)/no. of tested ticks.

b No. of infections/no. of tested ticks.

c No. of infections/no. of infected ticks.

d \% of the given pathogen species among the 113 infections.

e $B / T$ : includes infections reacting with this probe only; considered as belonging to the genus Babesia.

(Thomas and Mason, 1981) and T. taurotragi (Lawrence et al., 1994b) in Hy. rufipes and R. appendiculatus, respectively. Rhipicephalus e. evertsi appears as a very important vector for T. separata, T. equi, and Theileria sp. (sable) in South Africa. Interestingly, B. bigemina was detected in the salivary glands of one $R$. e. evertsi adult. The exact role of $R$. e. evertsi in the epidemiology of redwater remains unclear (De Vos and Potgieter, 1994). According to Büscher (1988), only nymphs transmit $B$. bigemina, and transovarial transmission does not occur. Further studies are needed to evaluate the role of $R$. e. evertsi adults in the circulation of $B$. bigemina.

To our knowledge, the remaining 23 vector-pathogen combinations were never described before. Among those, 11 involved combinations with multiple records at different sites. Three vector-pathogen combinations involved a tick species, $R$. gertrudae, that was never reported as a vector (Walker et al., 2000). One third of $R$. gertrudae specimens carried bacterial pathogens, all identified at species level. Four out of $6 \mathrm{~A}$. marginale infections were detected in this tick species suggesting a possible vector role of $R$. gertrudae in the epidemiology of gallsickness. In addition, E. ovina and A. centrale were each identified in one $R$. gertrudae. One vector-pathogen combination involved $T$. bicornis detected for the first time in ticks and previously reported in rhinoceroses (Nijhof et al., 2003; Govender et al., 2011), cattle (Muhanguzi et al., 2010), and nyala (Pfitzer et al., 2011). It was identified in the salivary glands of $26 R$. e. evertsi at 4 localities in the lowveld and in the highveld. This strongly suggests that $R$. e. evertsi transmits T. bicornis on a large area. Three vector-pathogen combinations involved $T$. buffeli which has recognized vectors in some parts of the world (Gubbels et al., 2000), but to our knowledge, no identified vector in South Africa. Theileria buffeli infections $(n=34)$ were detected in $R$. e. evertsi and $R$. (B.) decoloratus, as well as in 2 Hy. rufipes at sites situated in the lowveld and highveld. This suggests that $R$. e. evertsi and $R$. (B.) decoloratus

Table 5

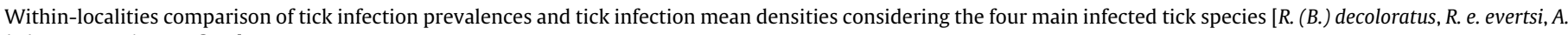
hebraeum, and Hy. rufipes].

\begin{tabular}{|c|c|c|c|c|c|c|}
\hline \multirow[t]{2}{*}{ Location } & \multicolumn{3}{|c|}{ Tick infection prevalence } & \multicolumn{3}{|c|}{ Tick pathogen mean density } \\
\hline & No. infected/tested & $z$-Values & $P$-Values & Mean infection/tick & $z$-Values & $P$-Values \\
\hline Sandveld (w) & $77 / 343(22.45 \%)$ & 4.02 & $<0.0001$ & $110 / 343(0.32)$ & 5.09 & $<0.0001$ \\
\hline Sandveld (d) & $24 / 251(9.56 \%)$ & & & $25 / 251(0.10)$ & & \\
\hline Willem Pretorius (w) & $14 / 137(10.22 \%)$ & 0.1 & 0.91 & $18 / 137(0.13)$ & 0.28 & 0.78 \\
\hline Willem Pretorius (d) & $25 / 254(9.84 \%)$ & & & $29 / 254(0.11)$ & & \\
\hline Tüssen-Die-Riviere (w) & $32 / 183(17.49 \%)$ & -0.55 & 0.58 & $33 / 183(0.18)$ & -0.09 & 0.93 \\
\hline Tüssen-Die-Riviere (d) & $21 / 108(19.44 \%)$ & & & $20 / 108(0.19)$ & & \\
\hline Bethal (w) & $2 / 3(66.67 \%)$ & 1.63 & 0.1 & $2 / 3(0.67)$ & 2.41 & 0.016 \\
\hline Bethal (d) & $3 / 62(4.84 \%)$ & & & $4 / 62(0.06)$ & & \\
\hline Thabazimbi (w) & $41 / 78(52.56 \%)$ & 4.51 & $<0.0001$ & $50 / 78(0.64)$ & 4.01 & $<0.0001$ \\
\hline Thabazimbi (d) & $10 / 80(12.50 \%)$ & & & $14 / 80(0.18)$ & & \\
\hline Lephalale (w) & $59 / 228(25.88 \%)$ & 2.43 & 0.01 & $71 / 228(0.31)$ & 2.54 & 0.01 \\
\hline Lephalale (d) & $21 / 131(16.3 \%)$ & & & $21 / 131(0.16)$ & & \\
\hline
\end{tabular}

$P$-Values are considered significant when below or equal to 0.05 . 
play a role in the maintenance of this parasite in South Africa. Three vector-pathogen combinations involved $A$. bovis and Theileria sp. (sable) identified in the salivary glands of tick species that were suspected vectors. Anaplasma bovis was reported by Tonetti et al. (2009) in entire $R$. e. evertsi ticks. Here, A. bovis was detected in the salivary glands of $11 R$. e. evertsi collected at 3 localities. Our observation corroborates previous reports and supports that $R$. e. evertsi may act as a vector for $A$. bovis. The detection of Theileria sp. (sable) in the salivary glands of $9 R$. (B.) decoloratus at 3 sites and of $5 \mathrm{~A}$. hebraeum at 2 of these sites indicates that these ticks may act as vectors in addition to $R$. e. evertsi and R. appendiculatus (Steyl et al., 2012). The observation of Theileria sp. (sable) in these 2 additional ticks contributes to explain the broad host range of Theileria sp. (sable) (Spitalska et al., 2005; Yusufmia et al., 2010; Pfitzer et al., 2011; Steyl et al., 2012). Babesia caballi has a known vector in South Africa, but it was observed in 2 new vector-pathogen combinations, involving a tick species known to transmit other pathogens. We detected B. caballi in the salivary glands of $7 R$. (B.) decoloratus at 3 locations suggesting that this tick may be an additional vector of $R$. e. evertsi (De Waal et al., 1988).

The remaining 12 vector-pathogen combinations involved only one or 2 records. Among those, 5 were previously suspected. This was the case for T. mutans in R. e. evertsi (De Vos and Roos, 1981), E. ovina in $R$. (B.) decoloratus (Schulz, 1940) and in Hy. rufipes (Hyalomma spp., Schulz, 1940), and T. taurotragi in H. rufipes (Binta et al., 1998). In addition, Babesia sp. (sable) (Oosthuizen et al., 2008) that was identified in the salivary glands of $H y$. rufipes is genetically very close to $B$. occultans (Oosthuizen et al., 2008) which is transmitted by Hy. rufipes (Thomas and Mason, 1981). Thus, our observation is not so surprising. The remaining 7 vector-pathogen combinations were unknown. Theileria bicornis was detected in one R. appendiculatus. Anaplasma bovis was detected in one R. (B.) decoloratus; its vector in South Africa is $R$. appendiculatus (Scott, 1994). Anaplasma platys which is generally associated with canine hosts (Huang et al., 2005) and $R$. sanguineus (Hoskins et al., 1991) was detected in one $R$. e. evertsi. Theileria sp. (giraffe) which was only known in giraffe (Oosthuizen et al., 2009) was detected in one $R$. e. evertsi and one $R$. (B) decoloratus. Finally, Theileria sp. (kudu), a pathogen of greater kudu (Nijhof et al., 2005) and nyala (T. angassii) (Pfitzer et al., 2011), and T. buffeli were identified in the salivary glands of $R$. appendiculatus. These 12 vector-pathogen combinations need further records for confirmation of the vector role of these ticks.

More than $70 \%$ of the collected tick species were attached to wild ruminants. Differences in infestation between wild and domestic hosts can be explained by a lower exposure to ticks of domestic animals that were treated with acaricides on a regular basis. Furthermore, domestic animals graze in managed camps restricted to several hectares while wild animal habitats are much larger and non-managed. Salivary glands of ticks that were attached to wild ruminants displayed significantly higher infection prevalences and pathogen mean densities than salivary glands of ticks attached to domestic animals at 3 localities. The pathogens that mainly contributed to the significant difference between wild and domestic animals were theilerial species transmitted only transstadially by the two-host tick R. e. evertsi. Rhipicephalus e. evertsi populations in reserves have a greater variety of hosts than those living on farms. This allows them to accumulate pathogen species originating from various hosts. This could be one explanation for the differences between salivary gland infections of ticks associated with wild vs. domestic ruminant environments. Another one could be that acaricide treatments are applied on domestic animals. This could affect the tick population structure (e.g. proportions between life stages) and therefore reduce vertical transmission and pathogen load in tick populations. Finally, differences in the susceptibility to acaricides of infected ticks and non-infected ticks could reduce the proportion of infected ticks in the environment. The frequence of given pathogen species in wild or domestic ruminants is also linked to the host preference of their vector ticks. For example, B. bovis transmitted by $R$. (B.) microplus in South Africa (Tonnesen et al., 2004 ) is almost exclusively found on cattle due to the fact that they represent the only effective hosts for $R$. (B.) microplus (Walker et al., 2003).

Here, $72 \%$ of the coinfections were observed in the two-host tick $R$. e. evertsi and only $22.6 \%$ in the one-host tick $R$. (B.) decoloratus, despite the fact that the one-host tick displayed a slightly higher infection prevalence and pathogen mean density. Tick life cycles with 2 and 3 hosts increase the chance of coinfections. Among the $529(23 \times 23)$ possibilities of coinfections, 6 pathogen associations involving 4 Theileria species and one Babesia species were significant. If coinfections in Ixodes ticks involving Borrelia spp., Babesia spp., Anaplasma spp., and Rickettsia spp. were described in North America (for example, Swanson et al., 2006) and Europe (for example, Lommano et al., 2012), much less is known on coinfections in ticks in southern Africa. Here, significant associations involved the most frequent pathogen species. This could indicate that associations are linked to pathogen frequency. However, the most frequent associations [Theileria sp. (sable) with $T$. separata, Theileria sp. (sable) with T. bicornis, and T. separata with T. bicornis] were the same as those observed in the blood of hosts exposed to these ticks (Berggoetz et al., 2013). This suggests that the associated species take advantage of their reciprocal presence. Future studies will have to understand the mechanisms of coinfections in ticks and evaluate their impact on wild and domestic ruminants.

The present study shows that many examined tick species were associated with a much broader pathogen range than previously known. Rhipicephalus e. evertsi and $R$. (B.) decoloratus are mainly concerned and appear as important vectors in South Africa. Pathogen species like T. bicornis, Theileria sp. (giraffe), Theileria sp. (kudu), and Babesia sp. (sable) were detected for the first time in ticks, more precisely in their salivary glands, which suggests their vector role. Furthermore, our observations showed that ticks from wild animal environments generally harboured more infections than ticks from domestic animal environments. This indicates that wild ruminants are more exposed to tick-borne pathogens which can probably be explained by the fact that they live in an uncontrolled environment with a higher host species richness than in the managed camp of domestic ruminants. Our results also shed light on the high frequency of coinfections in ticks.

\section{Acknowledgements}

We particularly thank F. Marais, J. Watson, and P. Nel from the Department of Economic Development, Tourism and Environmental affairs (Detea), Free State Province, for their technical and administrative support. Without their help this work would not have been possible. We thank the veterinarians E. Albertyn, F. Du Plessis, and N. Kriel for their help. We would like to thank all the Free State reserve managers for their support. We are grateful to the Webster family members for their help and kindness. We thank all farmers for welcoming us on their farms for tick collection. We address special thanks to R.A. Slobodeanu (Institute of Mathematics, University of Neuchâtel) for statistical analysis. We thank $\mathrm{O}$. Rais, V. Douet, and N. Tonetti for their useful advices in laboratory techniques, and A.S. Santos and S. Casati for positive controls. We would also like to thank «Le Réseau Ecologie des Interactions Durables» and the group "Tiques et Maladies à Tiques» as well as the "Ecole Doctorale», University of Neuchâtel. This study is part of the $\mathrm{PhD}$ thesis of M. Berggoetz. The Swiss National Science Foundation (SNSF) (FN 31003A_125492/1) funded this research. In addition, financial support was given by the Funds M. Wüthrich and A. 
Mathey-Dupraz (University of Neuchâtel) and the Swiss Academy of Sciences (SCNAT). We would like to thank the 2 reviewers for their useful comments.

\section{References}

Aktas, M., Altay, K., Dumanli, N., Kalkan, A., 2009. Molecular detection and identification of Ehrlichia and Anaplasma species in ixodid ticks. Parasitol. Res. 104 1243-1248.

Bekker, C.P.J., de Vos, S., Taoufik, A., Sparagano, O.A.E., Jongejan, F., 2002. Simultaneous detection of Anaplasma and Ehrlichia species in ruminants and detection of Ehrlichia ruminatium in Amblyomma variegatum ticks by reverse line blot hybridisation. Vet. Microbiol. 89, 223-238.

Berggoetz, M., Schmid, M., Ston, D., Wyss, V., Chevillon, C., Pretorius, A.-M., Gern, L., 2013. Tick-borne pathogens in the blood of wild and domestic ungulates in South Africa: interplay of game and livestock. Ticks Tick Borne Dis. (in press).

Bezuidenhout, J.D., Prozesky, L., du Plessis, J.L., van Amstel, S.R., 1994. Heartwater In: Coetzer, J.A.W., Thomson, G.R., Tustin, R.C. (Eds.), Infectious Diseases of Livestock, with Special Reference to Southern Africa. Oxford University Press, Oxford UK, pp. 351-370.

Binta, M.G., Losho, T., Allsopp, B.A., Mushi, E.Z., 1998. Isolation of Theileria taurotragi and Theileria mutans from cattle in Botswana. Vet. Parasitol. 77, 83-91.

Brothers, P.S., Collins, N.E., Oosthuizen, M.C., Bhoora, R., Troskie, M., Penzhorn, B.L. 2011. Occurrence of blood-borne tick-transmitted parasites in common tsessebe (Damaliscus lunatus) antelope in Northern Cape Province, South Africa. Vet. Parasitol. 183, 160-165

Büscher, G., 1988. The infection of various tick species with Babesia bigemina, its transmission and identification. Parasitol. Res. 74, 324-330.

Butler, C.M., Nijhof, A.M., Jongejan, F., van der Kolk, J.H., 2008. Anaplasma phagocytophilum infection in horses in the Netherlands. Vet. Rec. 162, 216-218.

Carmichael, I.H., Hobday, E., 1975. Blood parasites of some wild Bovidae in Botswana. Onderstepoort J. Vet. Res. 42, 55-62.

Clua, E., Buray, N., Legendre, P., Mourier, J., Planes, S., 2010. Behavioural response of sicklefin lemon sharks Negaprion acutidens to underwater feeding for ecotourism purposes. Marine Ecol. Prog. Ser. 414, 257-266.

De Vos, A.J., Roos, J.A., 1981. Observations on the transmission of Theileria mutans in South Africa. Onderstepoort J. Vet. Res. 48, 1-6.

De Vos, A.J., Potgieter, F.T., 1994. Bovine babesiosis. In: Coetzer, J.A.W., Thomson, G.R., Tustin, R.C. (Eds.), Infectious Diseases of Livestock, with Special Reference to Southern Africa. Oxford University Press, Oxford, UK, pp. 278-294.

De Waal, D.T., Potgieter, F.T., 1987. The transstadial transmission of Babesia caballi by Rhipicephalus evertsi evertsi. Onderstepoort J. Vet. Res. 54, 655-656.

De Waal, D.T., Van Heerden, J., Van den Berg, S.S., Stegmann, G.F., Potgieter, F.T., 1988. Isolation of pure Babesia equi and Babesia caballi organisms in splenectomized horses from endemic areas in South Africa. Onderstepoort J. Vet. Res. 55, 33-35.

De Waal, D.T., van Heerden, J., 1994. Equine babesiosis. In: Coetzer, J.A.W., Thomson, G.R., Tustin, R.C. (Eds.), Infectious Diseases of Livestock, with Special Reference to Southern Africa. Oxford University Press, Oxford, UK, pp. 295-304.

Georges, K., Loria, G.R., Riili, S., Greco, A., Caracappa, S., Jongejan, F., Sparagano, O., 2001. Detection of haemoparasites in cattle by reverse line blot hybridisation with a note on the distribution of ticks in Sicily. Vet. Parasitol. 99, 273-286.

Govender, D., Oosthuisen, M.C., Penzhorn, B.L., 2011. Piroplasm parasites of white rhinoceroses (Ceratotherium simum) in the Kruger National Park, and their relation to anaemia. J. South African Vet. Ass. 82, 36-40.

Gubbels, J.M., de Vos, A.P., van der Weide, M., Viseras, J., Schouls, L.M., de Vries, E., Jongejan, F., 1999. Simultaneous detection of bovine Theileria and Babesia species by reverse line blot hybridisation. J. Clin. Microbiol. 37, 1782-1789.

Gubbels, M.J., Hong, Y., van der Weide, M., Qi, B., Nijman, I.J., Guangyuan, L., Jongejan, F., 2000. Molecular characterisation of the Theileria buffeli/orientalis group. Int. J. Parasitol. 30, 943-952.

Hea, L., Fenga, H.H., Zhanga, W.J., Zhanga, Q.L., Fanga, R., Wanga, L.X., Tua, P., Zhoub, Y.Q., Zhaoa, J.L., Oosthuizend, M.C., 2011. Occurrence of Theileria and Babesia species in water buffalo (Bubalus babalis, Linnaeus, 1758) in the Hubei province, South China. Vet. Parasitol. 170, 323-326.

Horak, I.G., Williams, E.J., Van Schalkwyk, P.C., 1991. Parasites of domestic and wild animals in South Africa. XXV. Ixodid ticks on sheep in the north-eastern Orange Free State and in the Eastern Cape Province. Onderstepoort J. Vet. Res. $58,115-123$

Horak, I.G., Golezardy, H., Uys, A.C., 2007. Ticks associated with the three largest wild ruminants species in southern Africa. Onderstepoort J. Vet. Res. 74, 231-242.

Hoskins, J.D., 1991. Ehrlichial diseases of dogs: diagnosis and treatments. Canine Pract. 16, 13-21.

Huang, H., Unver, A., Perez, M.J., Orellana, N.G., Rikihisa, Y., 2005. Prevalence and molecular analysis of Anaplasma platys in dogs in Lara, Venezuela. Brazilian J. Microbiol. 36, 211-216.

Husson, F., Josse, J., Le, S., Mazet, J., 2012. FactoMineR: Multivariate exploratory data analysis and data mining with $R$. $R$ package version 1.20, http://CRAN.R-project.org/package=FactoMineR

Jacquiet, P., Dia, M.L., Perié, N.M., Jongejan, F., Uilenberg, G., Morel, P.C., 1990. Présence de Theileria annulata in Mauritania. Rev. Elev. Méd. Vét. Pays Trop. 43, 489-490.

Jansen, B.C., Neitz, W.O., 1956. The experimental transmission of Theileria ovis by Rhipicephalus evertsi. Onderstepoort J. Vet. Res. 27, 3-6.
Kaufmann, J., 1996. Parasitic Infections of Domestic Animals: a Diagnostic Manual. Birkhäuser Verlag, Basel, Schweiz, pp. 189.

Lawrence, J.A., de Vos, A.J., Irvin, A.D., 1994a. Theileria mutans infection. In: Coetzer J.A.W., Thomson, G.R., Tustin, R.C. (Eds.), Infectious Diseases of Livestock with Special Reference to Southern Africa. Oxford University Press, Oxford, UK, pp. 336-337.

Lawrence, J.A., de Vos, A.J., Irvin, A.D., 1994b. Theileria taurotragi infection. In: Coetzer, J.A.W., Thomson, G.R., Tustin, R.C. (Eds.), Infectious Diseases of Livestock, with Special Reference to Southern Africa. Oxford University Press, Oxford, UK, pp. 334-335.

Löhr, K.F., Meyer, H., 1973. Game anaplasmosis: the isolation of Anaplasma organisms from Antelope. Z. Tropenmed. Parasitol. 24, 192-197.

Löhr, K.F., Ross, J.P., Meyer, H., 1974. Detection in game of fluorescent and agglutination antibodies to intraerythrocytic organisms. Z. Tropenmed. Parasitol. 25, 217-226.

Lommano, E., Bertaiola, L., Dupasquier, C., Gern, L., 2012. Infections and coinfections of questing Ixodes ricinus ticks by emerging zoonotic pathogens in western Switzerland. Appl. Environ. Microbiol. 78, 4606-4612.

Matthysse, J.G., Colbo, M.H., 1987. The Ixodid Ticks of Uganda: Together with Species Pertinent to Uganda Because of Their Present Distribution. Entomological Society of America, College Park, MD.

Matjila, P.T., Penzhorn, B.L., Bekker, C.P.J., Nijhof, A.M., Jongejan, F., 2004. Confirmation of the occurrence of Babesia canis vogeli in domestic dogs in South Africa. Vet. Parasitol. 122, 119-125.

Muhanguzi, D., Matovu, E., Waiswa, C.,2010. Prevalence and characterization of Theileria and Babesia species in cattle under different husbandry systems in Western Uganda. Int. J. Anim. Vet. Adv. 2, 51-58.

Nagore, D., Garcia-Sanmartin, J., Garcia-Perez, A.L., Juste, R.A., Hurtado, A. 2004. Identification, genetic diversity and prevalence of Theileria and Babesia species in sheep population from northern Spain. Int. J. Parasitol. 34, 1059-1067.

Neitz, W.O., Du Toit, P.J., 1932. Bovine anaplasmosis. A method of obtaining pure strains of Anaplasma marginale and A. centrale by transmission through antelopes. In: 18th Report of the Director of Veterinary Services and Animal Industry, Pretoria, Union of South Africa, pp. 3-20.

Neitz, W.O., 1935. Bovine anaplasmosis. The transmission of Anaplasma marginale to black wildebeest (Connochaetes gnou). Onderstepoort J. Vet. Res. Sc. An. Ind. 5, 9-11.

Neitz, W.O., 1956. A consolidation of our knowledge of the transmission of tick-borne diseases. Onderstepoort J. Vet. Res. 27, 115-163.

Nijhof, A.M., Penzhorn, B.L., Lynen, G., Mollel, J.O., Morkel, P., Bekker, C.P.J., Jongejan, F., 2003. Babesia bicornis sp. nov. and Theileria bicornis sp. nov.: tick-borne parasites associated with mortality in the black rhinoceros (Diceros bicornis). J. Clin. Microbiol. 41, 2249-2254.

Nijhof, A.M., Pillary, V., Steyl, J., Prozesky, L., Stoltsz, W.H., Lawrence, J.A., Penzhorn, B.L., Jongejan, F., 2005. Molecular characterization of Theileria species associated with mortality in four species of African antelopes. J. Clin. Microbiol. 43, 5907-5911

Norval, R.A.I., 1975. Studies on the ecology of Haemaphysalis silacea Robinson 1912 (Acarina: Ixodidae). J. Parasitol. 61, 730-736

Oosthuizen, M.C., Zweygarth, E., Collins, N.E., Troskie, M., Penzhorn, B.L., 2008. Identification of a novel Babesia sp. from a sable antelope (Hippotragus niger Harris 1838). J. Clin. Microbiol. 46, 2247-2251.

Oosthuizen, M.C., Allsopp, B.A., Troskie, M., Collins, N.E., Penzhorn, B.L., 2009. Identification of novel Babesia and Theileria species in South African giraffe (Giraffa camelopardalis, Linnaeus, 1758) and roan antelope (Hippotragus equinus, Desmarest 1804). Vet. Parasitol. 163, 39-46.

Oura, C.A.L., Bishop, R.P., Wampande, E.M., Lubega, G.W., Tait, A., 2004. Application of reverse line blot assay to the study of haemoparasites in cattle in Uganda. Int J. Parasitol. 34, 603-613.

Pfitzer, S., 2009. Occurrence of tick-borne haemoparasites in nyala (Tragelaphus angasii) in KwaZulu-Natal and Eastern Cape Province, South Africa. M.SC. Thesis. University of Pretoria.

Pfitzer, S., Oosthuizen, M.C., Bosman, A.M., Vorster, I., Penzhorn, B.L., 2011. Tickborne parasites in nyala (Tragelaphus angasii, Gray 1849) from KwaZulu-Natal South Africa. Vet. Parasitol. 176, 126-131.

Pipano, E., 1994. Theileria annulata theileriosis, In: Coetzer, J.A.W. Thomson, G.R., Tustin, R.C. (Eds.), Infectious Diseases of Livestock, with Special Reference to Southern Africa. Oxford University Press, Oxford, UK, pp. 341-348.

Potgieter, F.T., Els, H.J., 1977. Light and electron microscopic observations on the development of Babesia bigemina in larvae, nymphae and non-replete females of Boophilus decoloratus. Onderstepoort J. Vet. Res. 44, 213-232.

Potgieter, F.T., 1981. Tick transmission of anaplasmosis in South Africa. In: Proceedings of the International Conference on Tick Biology and Control, pp. 53-56.

Raup, D.M., Crick, R.E., 1979. Measurement of faunal similarity in paleontology. J. Paleontol. 53, 1213-1227.

Ros-Garcia, A., M'Ghirbi, Y., Bouattour, A., Hurtado, A., 2011. First detection of Babesia occultans in Hyalomma ticks from Tunisia. Parasitology 138, 578-582.

Schnittger, L., Yin, H., Qi, B., Gubbels, M.J., Beyer, D., Niemann, S., Jongejan, F., Ahmed, J.S., 2004. Simultaneous detection and differentiation of Theileria and Babesia parasites infecting small ruminants by reverse line blotting. Parasitol. Res. 92, 189-196.

Schulz, K., 1940. A rickettsiosis new to South Africa. Onderstepoort J. Vet. Sc. An. Ind. 13, 287-289. 
Scott, G.R., 1994. Lesser-known rickettsias infecting livestock. In: Coetzer, J.A.W., Thomson, G.R., Tustin, R.C. (Eds.), Infectious Diseases of Livestock, with Special Reference to Southern Africa. Oxford University Press, Oxford, UK, pp. 371-377.

Skaug, H., Fournier, D., Nielsen, A., 2010. Glmm ADMB-package, http://127.0.0.1:13596/library/glmmADMB/html/glmmADMB-package.html

Spitalska, E., Riddell, M., Heyne, H., Sparagano, O.A.E., 2005. Prevalence of theileriosis in red hartebeest (Alcelaphus buselaphus caama) in Namibia. Parasitol. Res. 97, 77-79.

Steyl, J.C.A., Prozesky, L., Stoltsz, W.H., Lawrence, J.A., 2012. Theileriosis (Cytauxzoonosis) in roan antelope (Hippotragus equinus): Field exposure to infection and identification of potential vectors. Onderstepoort J. Vet. Res. 70, $1-8$.

Swanson, S.J., Neitzel, D., Reed, K.D., Belongia, E.A., 2006. Coinfections acquired from Ixodes ticks. Clin. Microbiol. Rev. 19, 708-727.

Thomas, S.E., Mason, T.E., 1981. Isolation and transmission of an unidentified Babesia sp. infective for cattle. Onderstepoort J. Vet. Res. 48, 155-158.
Tonetti, N., Berggoetz, M., Rühle, C., Pretorius, A.M., Gern, L., 2009. Ticks and tickborne pathogens from wildlife in the Free State province, South Africa. J. Wildl. Dis. $45,437-446$.

Tonnesen, M.H., Penzhorn, B.L., Bryson, N.R., Stoltsz, W.H., Masibigiri, T., 2004. Displacement of Boophilus decoloratus by B. microplus in the Soutpansberg region, Limpopo province, South Africa. Exp. Appl. Acarol. 32, 199-208.

Walker, J.B., 1991. A review of the ixodid ticks (Acari, Ixodidae) occurring in Southern Africa. Onderstepoort J. Vet. Res. 58, 81-105.

Walker, J.B., Keirans, J.E., Horak, I.G. (Eds.), 2000. The genus Rhipicephalus (Acari, Ixodidae). Cambridge University Press, Cambridge, UK.

Walker, A.R., Bouattour, A., Camicas, J.-L., Estrada-Peña, A., Horak, I.G., Latif, A.A., Pegram, R.G., Preston, P.M. (Eds.), 2003. Ticks of Domestic Animals in Africa: a Guide to Identification of Species. , Bioscience Reports, Edinburgh.

Yusufmia, S.B.A.S., Collins, N.E., Nkuna, R., Troskie, M., Van den Bossche, P., Penzhorn, B.L., 2010. Occurrence of Theileria parva and other haemoprotozoa in cattle at the edge of Hluhluwe-iMfolozi Park, KwaZulu-Natal, South Africa. J. South African Vet. Ass. 81, 45-49. 